\title{
BMJ Open Parent and caregiver perspectives on home-based newborn care in low-income settings: protocol for a systematic review of qualitative studies
}

\author{
Alessandra N Bazzano, Erica Felker-Kantor, Aiko Kaji, Lisa Saldanha
}

To cite: Bazzano AN, FelkerKantor E, Kaji A, et al. Parent and caregiver perspectives on home-based newborn care in low-income settings: protocol for a systematic review of qualitative studies. BMJ Open 2016;6:e012137. doi:10.1136/bmjopen-2016012137

- Prepublication history and additional material is available. To view please visit the journal (http://dx.doi.org/ 10.1136/bmjopen-2016012137).

Received 4 April 2016 Revised 19 July 2016 Accepted 25 July 2016

CrossMark

Department of Global Community Health and Behavioral Sciences, Tulane University School of Public Health and Tropical Medicine, New Orleans, Louisiana, USA

Correspondence to Dr Alessandra N Bazzano; abazzano@tulane.edu

\section{ABSTRACT}

Introduction: Newborn health and survival are closely linked to essential newborn care provided within the first days and weeks of an infant's life by parents and caregivers at home and within the community. Newborn care practices are often socially and culturally determined and have been explored in qualitative and formative research related to improving neonatal survival. We aim to provide a comprehensive review of qualitative studies on parent and caregiver experiences of newborn care practices with a view to identifying barriers and facilitators that may impact on newborn health. The rationale is that providing this information will be useful for intervention design and programme scale up for newborn survival.

Methods and analysis: We will systematically review qualitative studies reporting on newborn care practices. The Enhancing Transparency in Reporting the Synthesis of Qualitative Research (ENTREQ) statement will be used for reporting the stages of the review and dissemination. The search period will include all studies published from 2006 to 2016. Study selection will incorporate the ENTREQ and Preferred Reporting Items for Systemic Reviews and Meta-Analyses (PRISMA) guidelines and quality assessment will be completed using Critical Appraisal Skills Programme (CASP) guidelines. Pending the identification of sufficient data of good quality, we will conduct a full synthesis of the studies identified by the review.

Ethics and dissemination: The results will be disseminated through peer-reviewed publications, conference presentation and directly to organisations involved in newborn health. Formal ethical approval from the author's institution is not required, as no primary data or identifying data will be collected. Trial registration number: CRD42016035674.

\section{BACKGROUND}

Community-based or home-based care of the newborn infant is a crucial component of survival, healthy growth and optimal development for all children. ${ }^{1}$ Following delivery, parents and family caregivers play the most

\section{Strengths and limitations of this study}

- Focus on lower income countries.

- Synthesis of qualitative findings where currently none exists in the published literature.

- English language studies only will be included.

- Potential for missing material that may be relevant but is not found by the search strategy.

important role in protecting and providing for newborns in the most vulnerable period of their young lives, the 28 days following birth. $^{2}$ Despite strong evidence for the effectiveness of feasible interventions to reduce newborn mortality, ${ }^{3}$ which continues to be unacceptably high, ${ }^{4}$ coverage of these interventions is low. ${ }^{5}$

In evaluating research priorities for improving newborn health and birth outcomes, researchers and key stakeholders have identified a significant number of domains related to caregiver perceptions and behaviours, and related to home and community newborn care practices. ${ }^{6}$ Providing data on these prioritised research topics is key for scale up of coverage and effective implementation of interventions aimed at improving newborn health.

Our rationale in conducting this review is that while many individual qualitative and formative research studies have been conducted on newborn care practices in the home and community, ${ }^{7-10}$ to date there has not been a systematic review or synthesis of the existing qualitative research. Conducting a systematic review will provide comprehensive and useful data for programming and policy related to facility and community care for newborns, as well as guidance for intervention design and scale up of existing programmes.

Newborn care practices comprise a multifaceted group of behaviours, thus qualitative 
methodologies and the data obtained from qualitative research are especially appropriate for gaining information about these practices, particularly in low-income countries where such behaviours will vary based on the sociocultural context. ${ }^{9}$

The primary objective of the proposed study is to systematically review qualitative literature related to newborn care practices with a focus on parent and caregiver perceptions and experiences in low-income settings, focusing on information related to barriers and facilitators that may affect interventions for newborn survival.

\section{METHODS AND ANALYSIS}

This systematic review has been registered with the International Prospective Register of Systematic Reviews (PROSPERO): registration number CRD42016035674.

\section{Study design}

The review to be undertaken will follow the Enhancing Transparency in Reporting the Synthesis of Qualitative Research (ENTREQ) statement in reporting the stages of the review and dissemination. In view of the unique nature of qualitative research, the review will employ the ENTREQ guidelines ${ }^{11}$ and the Preferred Reporting Items for Systemic Reviews and Meta-Analyses (PRISMA) guidelines, as the latter is more closely related to reviews of quantitative literature and may not be sufficient alone. $^{12}$

Studies will be included where data are presented as having been directly obtained from participants who are parents or caregivers of newborns (infants under 28 days of age, including low birthweight or small babies), whether born at home or at a facility, with or without skilled attendance. Caregivers will be defined as mothers/fathers or other adult family or community members who provide day to day physical and psychological support to meet the basic needs of newborn infants. Community health workers will not be considered as caregivers for the purposes of this review, though we acknowledge that they may be involved in caring for newborn infants at specific points in time.

Studies will be included if they use widely accepted qualitative data collection methods (interviews, focus groups, direct observation, participatory action research, etc) and analysis methods. Studies involving mixed methods where the qualitative data will be difficult to extract will be excluded, as will studies with heterogeneous participant groupings or studies with settings where perceptions of parents/caregivers cannot be extracted. Commentaries will not be included. Additionally, studies from countries other than those defined by the World Bank as low-income and lower-middle-income countries will be excluded. ${ }^{13}$

For the purpose of this systematic review, newborn care practices will be defined as all actions taken by parents/caregivers that provide for the essential biological, physiological and psychological needs of the newborn infant following delivery up to 28 days of life. These will include, but are not limited to, the essential newborn care practices as defined in the international reference literature ${ }^{14}$ such as cord care, drying and wrapping after delivery, initiation of breast feeding, bathing, thermal control, breast feeding and care seeking for newborn illness.

Given that newborn mortality is highest in areas of low socioeconomic status and with poor health infrastructure, ${ }^{2} 15$ only studies from low-income and lower-middleincome settings, as defined by World Bank, will be included.

\section{Search strategy}

The following electronic databases which are considered to be the most relevant for the topic will be searched: MEDLINE (PubMed), Embase and Cumulative Index to Nursing and Allied Health Literature (CINAHL) through EBSCO. The initial search strategy will be developed for MEDLINE and then adapted for other databases. Medical subject headings $(\mathrm{MeSH})$ will initially be used, followed by free-text terms using controlled vocabulary (see online supplementary annex 1 for a detailed description of the search strategy). A library and information scientist specialised in public health will assist in further piloting of search strategies, will finalise the search strategy and will perform the database searches for the review.

Results will be restricted to English language publications from the last 10 years. In addition to the aforementioned search strategy, we will manually search reference lists of included studies to identify any additional studies that fit the inclusion criteria. Experts working in the field may also be contacted to identify relevant literature that has not been obtained through the database and manual search of reference lists. Results from these searches will again be limited to publications in English from the last 10 years.

\section{Study selection}

Search results will be imported into EndNote software (Thomson Reuters (Scientific) LLC). Duplicates and irrelevant studies will be removed. Two independent reviewers will first screen study titles and abstracts for eligibility. Eligibility will be tested against predetermined inclusion criteria and quality assessment guidelines. Three EndNote folders will be created: one for studies that meet initial search criteria (where agreed by both reviewers), one for studies that do not meet criteria (where agreed by both reviewers) and one for further full-text review to determine eligibility. In all cases, the decision to include or exclude a study must be agreed on by both reviewers. If a decision cannot be reached, a third reviewer will make the final decision.

A flow diagram using PRISMA guidelines for reporting of systematic reviews will be used in reporting of the selection process and results. ${ }^{16}$ 


\section{Quality appraisal}

To improve the internal validity of the review, each study will be assessed for quality according to the Critical Appraisal Skills Programme (CASP) checklist. ${ }^{17}$ Studies must meet minimum objective criteria to be considered of sufficient quality. The criteria will include domains such as appropriateness of study design, sampling methodology, as well as data collection techniques and analysis methods used in each study. The authors will document studies that were excluded on the basis of quality. Descriptive information of these studies will be available if requested but will not be included in the review nor synthesis of findings.

Two reviewers will independently review each study against the checklist to reach consensus. In cases of nonconsensus, a third reviewer will decide the outcome. A quality assessment table will be created to facilitate comparisons among the reviewed studies.

\section{Data extraction}

Specific characteristics from included studies will be extracted and complied into a unified data matrix. A single reviewer will complete abstract review and a second reviewer will check for accuracy. Extracted data will include, but not be limited to, reference details (author/data/publication), methodological approach (eg, interviews/focus groups), conceptual theory underlying the study (eg, grounded theory), objectives or aims of the study, sampling methodology, sociodemographic characteristics of participants, country/region and analysis method.

\section{ANALYSIS}

The final analysis plan will be dependent on the results of the review. If results are relevant and meet the stated objectives, data from the Results, Discussion and Conclusion sections of included studies will be extracted into NVivo V.11 software (NVivo qualitative data analysis software, V.11, 2015; QSR International Pty.) for further synthesis. Thematic analysis, whereby themes that are descriptive of the data will be developed, analysed and presented, will be conducted. Tables and visual representations of the thematic analysis will be provided.

\section{DISCUSSION}

To our knowledge, this will be the first study to systematically review and synthesise qualitative data on newborn care practices in low-income countries from the perspective of caregivers. The focus on qualitative findings will allow for rich data on complex and often heterogeneous care practices in lower income countries where newborn mortality is most prevalent to be made more widely available. The findings will provide insight into the barriers and facilitators that hinder or enable implementation of newborn care best practices.
Contributors ANB was responsible for the overall conception and design of the work and drafted and revised it for important intellectual content. EF-K also drafted and revised for important intellectual content. AK and LS assisted with the acquisition, analysis and interpretation of data for the work. All authors reviewed and agreed on final approval of the version to be published. All authors have agreed to be accountable for all aspects of the work in ensuring that questions related to the accuracy or integrity of any part of the work are appropriately investigated and resolved.

Competing interests None declared.

Provenance and peer review Not commissioned; externally peer reviewed.

Data sharing statement The authors will make any additional unpublished data freely available to anyone who requests it.

Open Access This is an Open Access article distributed in accordance with the Creative Commons Attribution Non Commercial (CC BY-NC 4.0) license, which permits others to distribute, remix, adapt, build upon this work noncommercially, and license their derivative works on different terms, provided the original work is properly cited and the use is non-commercial. See: http:// creativecommons.org/licenses/by-nc/4.0/

\section{REFERENCES}

1. Bhutta ZA, Darmstadt GL, Hasan BS, et al. Community-based interventions for improving perinatal and neonatal health outcomes in developing countries: a review of the evidence. Pediatrics 2005;115(2 Suppl):519-617.

2. Lawn JE, Blencowe H, Oza S, et al. Every Newborn: progress, priorities, and potential beyond survival. Lancet 2014;384:189-205.

3. Darmstadt GL, Marchant T, Claeson M, et al. A strategy for reducing maternal and newborn deaths by 2015 and beyond. BMC Pregnancy Childbirth 2013;13:216.

4. Liu L, Johnson HL, Cousens S, et al. Global, regional, and national causes of child mortality: an updated systematic analysis for 2010 with time trends since 2000. Lancet 2012;379:2151-61.

5. Bhutta ZA, Das JK, Bahl R, et al. Can available interventions end preventable deaths in mothers, newborn babies, and stillbirths, and at what cost? Lancet 2014;384:347-70.

6. Yoshida S, Rudan I, Lawn J, et al. Newborn health research priorities beyond 2015. Lancet 2014;384:e27-9.

7. Waiswa P, Namazzi G, Kerber K, et al. Designing for action: adapting and implementing a community-based newborn care package to affect national change in Uganda. Glob Health Action 2015;8:24250.

8. Hill Z, Manu A, Tawiah-Agyemang C, et al. How did formative research inform the development of a home-based neonatal care intervention in rural Ghana? J Perinatol 2008;28(Suppl 2):S38-45.

9. Neonatal Mortality Formative Research Working Group. Developing community-based intervention strategies to save newborn lives: lessons learned from formative research in five countries. $J$ Perinatol 2008;28 2):S2-8.

10. Syed U, Khadka N, Khan A, et al. Care-seeking practices in South Asia: using formative research to design program interventions to save newborn lives. J Perinatol 2008;28(Suppl 2):S9-13.

11. Tong A, Flemming K, Mclnnes $\mathrm{E}$, et al. Enhancing transparency in reporting the synthesis of qualitative research: ENTREQ. BMC Med Res Methodol 2012;12:181.

12. Fleming PS, Koletsi D, Pandis N. Blinded by PRISMA: are systematic reviewers focusing on PRISMA and ignoring other guidelines? PLOS ONE 2014;9:e96407.

13. Country and Lending Groups. http://data.worldbank.org/about/ country-and-lending-groups.

14. World Health Organization. Pregnancy, Childbirth, Postpartum and Newborn Care: A Guide for Essential Practice. 3rd edn. Geneva: World Health Organization, 2015.

15. Enweronu-Laryea C, Dickson KE, Moxon SG, et al. Basic newborn care and neonatal resuscitation: a multi-country analysis of health system bottlenecks and potential solutions. BMC Pregnancy Childbirth 2015;15(Suppl 2):S4.

16. Moher D, Liberati A, Tetzlaff J, et al. Preferred reporting items for systematic reviews and meta-analyses: the PRISMA statement. PLOS Med 2009;6:e1000097.

17. (CASP) CASP. Qualitative research checklist. In: Edited by CASP Checklists. Oxford, 2014. 